\title{
Special issue based on selected ONDM 2014 papers
}

\author{
Paolo Monti ${ }^{1}$ - Piero Castoldi ${ }^{2}$ - Lena Wosinska ${ }^{1}$
}

Published online: 19 May 2015

(C) Springer Science+Business Media New York 2015

The 18th International Conference on Optical Networks Design and Modeling-ONDM 2014 (http://ondm2014.ict. kth.se) - was held in Stockholm, Sweden, on May 19-22, 2014.

ONDM is one of the premier venues in Europe for the optical network community to meet and exchange ideas. Following on the tradition set by its past editions, ONDM 2014 addressed cutting-edge research in optical networks, as well as topics where optical networks meet other areas and disciplines, such as software- defined networking (SDN), fixed-mobile convergence (FMC), and future internet design.

ONDM 2014 was a three and a half day event organized by the Optical Networks Laboratory (ONLab) in the School of Information and Communication Technology, KTH Royal Institute of Technology. ONDM 2014 was technically cosponsored by IFIP TC 6 (working group 6.10), by the IEEE Photonics Society, and by the IEEE Communication Society with the endorsement of TAOS and ONTC. The conference was also supported by Ericsson AB, the IEEE Swedish Chapter, the FP7 project DISCUS, the Swedish Research Council, and the City of Stockholm.

More than 100 high-quality technical papers were submitted by researchers from 30 countries all over the world.

Paolo Monti

pmonti@kth.se

Piero Castoldi

castoldi@sssup.it

Lena Wosinska

wosinska@kth.se

1 School of Information and Communication Technology, KTH Royal Institute of Technology, 16440 Kista, Sweden

2 Istituto di Tecnologie della Comunicazione, dell'Informazione e della Percezione, Scuola Superiore Sant'Anna, 56127 Pisa, Italy
All submitted papers were peer-reviewed by the Members of the Technical Program Committee and by external reviewers. ONDM 2014 had a rich program that included also a workshop of the FP7 project DISCUS (The DIStributed Core for unlimited bandwidth supply for all Users and Services). The 40 accepted contributions together with 10 invited papers and 3 keynote talks were organized in 10 oral and 1 poster sessions. The authors of the accepted papers belong to 22 nations from Europe, the Americas, Africa, the Middle, and the Far East. Overall, 20 countries were represented at the conference by the 108 delegates that attended the event.

Based on comments received from the reviewers, the authors of nine accepted papers were invited to submit an extended version of their work for possible publication in this Special Issue of the Springer Photonic Network Communications (PNET) journal. After a thorough review process, eight invited papers have been selected for publication. In addition, this Special Issue includes an invited paper that went through the same review process of the other accepted papers. A brief summary of the accepted papers is provided next.

In "Optical Transport and Challenges in the Networked Society" B. Skubic, P. Öhlén, A. Rostami, Z. Ghebretensaé, N. Lindqvist, and K. Laraqui describe the challenges and role played by optical transport in the future Networked Society. This position paper covers the role of optics in providing a transport solution for $5 \mathrm{G}$ radio, in addition to describing the impact of integrated photonics in providing low-cost Dense Wavelength Division Multiplexing (DWDM) solutions which could serve as the basis for $5 \mathrm{G}$ transport satisfying requirements on performance, low cost and flexibility.

In "Orchestrating Connectivity Services to support Elastic Operations in Datacenter Federations" A. Asensio, M. Ruiz, and L. Velasco propose a strategy to provision interdatacenter connectivity services using the data center federation idea. Datacenter federation(s) is a concept by which 
small independently operated datacenter (DC) infrastructures increase their revenue by using IT resources that would otherwise be under-utilized and expand their geographic coverage without building new DCs. The authors show that by using their proposed strategy it is possible to save up to $20 \%$ of the energy costs and more than $40 \%$ of communication costs.

In "Demonstration of a Novel Software-Defined FlexPON" L. Zhou, G. Peng, and N. Chand propose and experimentally assess the performance of a novel full service software-defined flexible Passive Optical Network (FlexPON) solution that has three innovative features: (1) digital signal processing (DSP)-enabled flexible transceiver and flexible link, (2) optical line terminal (OLT) side access network resource virtualization, and (3) software-defined programmable network functions and resource scheduling.

In "Strategies for Optical Transport Network Recovery under Epidemic Network Failures" S. Ruepp, A. M. Fagertun, and V. Kosteas evaluate two rerouting strategies and propose four policies for failure handling in a connection-oriented optical transport network with a Generalized MultiProtocol Label Switching control plane. They show that source rerouting outperforms on-site rerouting and that there exists a clear trade-off between policy performance and network resource consumption, which must be addressed by network operators while improving the robustness of their transport infrastructures.

In "Spectrum Fragmentation Issue in Flexible Optical Networks Analysis and Good Practices" D. Amar, E. Le Rouzic, N. Brochier, J.-L. Auge, C. Lepers, and N. Perrot present an exhaustive analysis and an accurate evaluation of the spectrum fragmentation issue in flexible optical networks. They also propose new metric for fragmentation measurements and some approaches to address the fragmentation problem.

In "Routing, Modulation, Spectrum and Launch Power Assignment to Maximize the Traffic Throughput of a Nonlinear Optical Mesh Network” D. J. Ives, P. Bayvel, and S. J. Savory investigate the optimization of routing, modulation format adaptation, spectral and launch power assignment as a mean of improving the utilization of limited network resources and increasing the network throughput. They show, in the three test networks, that the launch power allocation and channel spectral assignment can improve the transmission SNR margin over the fixed modulation, fixed power, fully load link worst case by approximately $3-4 \mathrm{~dB}$.

In "Dynamic Connection Establishment and Network Reoptimization in Flexible Optical Networks" P. Soumplis, K. Christodoulopoulos, and E. Varvarigos present an algorithm that when the spectrum is fragmented, i.e., a connection establishment is blocked, reactively re-optimizes the network by shifting in the spectrum domain and/or rerouting existing connections. Their simulation results show that the blocking probability can be substantially reduced using their proposed techniques as opposed to a network that does not reactively defragments the spectrum.

In "Optical Wireless Network Convergence in Support of Energy Efficient Mobile cloud Services" M. P. Anastasopoulos, A. Tzanakaki, B. Rofoee, S. Peng, Y. Yan, D. Simeonidou, G. Landi, G. Bernini, N. Ciulli, J.F. Riera, E. Escalona, K. Katsalis propose an infrastructure and architectural approach based on the orchestrated planning and operation of optical data centre and wireless access networks. To this end, they present a novel formulation based on a multi-objective nonLinear programming model that considers energy-efficient virtual infrastructure planning over the converged wireless, optical network interconnecting DCs with mobile devices, taking a holistic view of the infrastructure. Their results identify trends and trade-offs related to end-to-end service delay, mobility, resource requirements, and energy consumption levels of the infrastructure across the various technology domains.

In "Optimal Design for p-Cycle Protected Elastic Optical Networks" Y. Wei, K. Xu, Y. Jiang, H. Zhao, and G. Shen study, in elastic optical networks, the advantages of p-Cycle-based network protection techniques as opposed to the ring cover technique. The authors develop Integer Linear Programming (ILP) models to minimize the required protection capacity and the used link spectra in the whole network. The result presented in the paper show that the p-Cycle technique allows for lower spare capacity redundancy than the ring cover technique.

\section{ONDM 2014 General Chair}

Lena Wosinska, KTH Royal Institute of Technology, Sweden,wosinska@kth.se

\section{ONDM 2014 TPC Co-Chairs}

Paolo Monti, KTH Royal Institute of Technology, Sweden, pmonti@kth.se

Piero Castoldi, Scuola Superiore S.Anna, Italy, castoldi@sssup.it

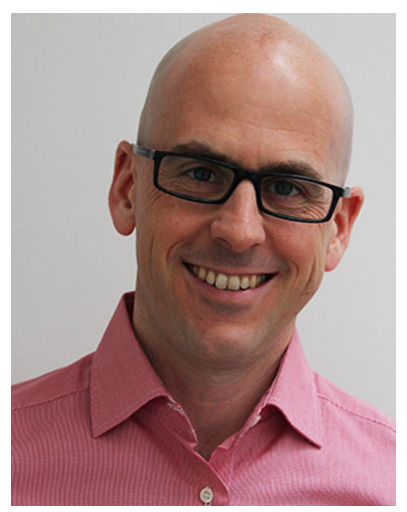

Paolo Monti received a $\mathrm{Ph} . \mathrm{D}$. in Electrical Engineering (2005) from The University of Texas at Dallas (UTD). From 2006 to 2008, he worked as a Research Associate of the Open Networking Advance Research (OpNeAR) Lab at UTD. He joined KTH Royal Institute of Technology in September 2008 where he is currently a researcher and the deputy director of the Optical Networks Laboratory (ONLab). He published more than 100 papers in peer-reviewed international journals and conferences. Dr. Monti is serving on the editorial boards of the IEEE Journal on Selected Areas in Communications- 
Series on Green Communications and Networking and of the Springer Photonic Network Communications journal. He was one of the Guest Editors of the Special Issue on "Green Networking and Computing" of the Journal of High Speed Networks, and he is the leading editor of a special issue of the Springer Photonic Network Communications journal on "Green Optical Networks". Dr. Monti regularly participates in several TPCs including IEEE Globecom and ICC where he also co-chaired a number of workshops on WDM network survivability (ICC 2012) and green broadband access (ICC 2013, Globecom 2014, ICC 2015). Dr. Monti was the TPC co-chair of ONDM 2014 and served/serves as TPC co-chair for the Symposium on Optical and Grid Computing in IEEE ICNC 2014 and ICNC 2016. His main research interests are within the networking aspects of all-optical networks, while part of his work recently focused on the architectural and control challenges posed by next-generation mobile communication (i.e., 5G) on optical transport networks. Dr. Monti is a member of the IEEE Communication Society.

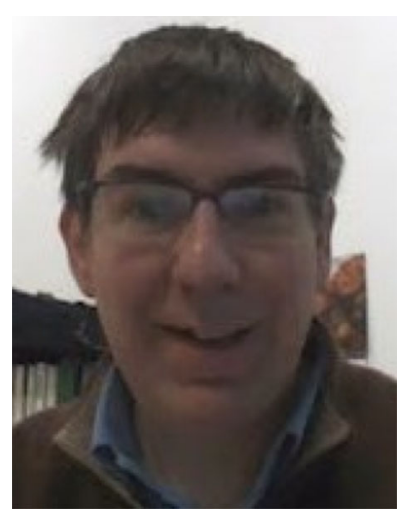

Piero Castoldi has been Associate Professor at Scuola Superiore Sant'Anna, Pisa, Italy since 2001. He is also Head of the "Networks and Services" Area at the Institute of Communication, Information and Perception Technologies (TeCIP) of Scuola Superiore Sant'Anna. Formerly, he has been Coordinator of "Communication" MacroArea at the TeCIP Institute. He has also served as Project Manager of many projects of the Inter-universitary National Consortium for Telecommunications (CNIT), and he has been Director of the National Laboratory of Photonic Networks in the period 2005-2010. He holds a Master degree cum laude in Electrical Engineering from the University of Bologna (Italy) and a Ph.D. degree in Information Technology from the University of Parma (Italy). He has also been visiting Postdoc and Researcher at the Department of Electrical Engineering at Princeton University (USA) for a total of 18 months in the period 1996-2000. He had leading roles in the following EU projects: BONE, STRONGEST, IDEALIST, OFELIA, and FED4FIRE. His research interests cover telecommunications networks architectures, in particular control of optical networks, traffic engineering and reliability, and data center architectures for grids and clouds. He is author of more than 300 publications in international journals and conference proceedings, in addition to a single author of a book on CDMA. He has also filed a total of 20 patents. Prof. Castoldi is a Senior Member of IEEE.

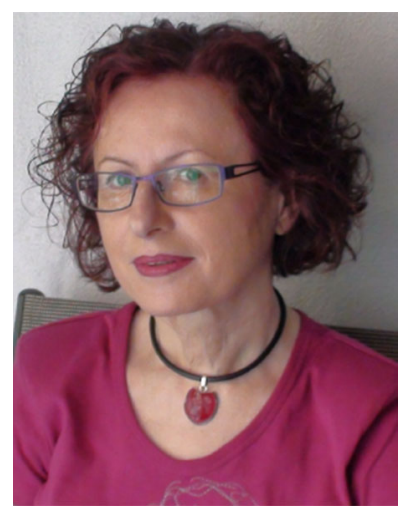

Lena Wosinska received her Ph.D. degree in Photonics and Docent degree in Optical Networking from the Royal Institute of Technology (KTH) in Stockholm, Sweden, where she is currently a Full Professor in Telecommunication in the School of Information and Communication Technology (ICT). She is founder and leader of the Optical Networks Lab (ONLab) at KTH/ICT. She has been working in several EU projects and coordinating a number of national and international research projects. Her research interests include fiber access networks, energy-efficient optical networks, photonics in switching, optical network management, reliability and survivability. She has been involved in many professional activities including guest editorship of OSA, Elsevier and Springer journals, serving in TPC of several conferences, as well as reviewer for many journals and project proposals. In 2007-2009, she was an Associate Editor of OSA Journal of Optical Networking, and in 2009-2013, she has been Associate Editor of IEEE/OSA Journal of Optical Communications and Networking. Currently, she is serving on the Editorial Board of Springer Photonic Networks Communication Journal. She was a co-chair of SPIE APOC 2008 and the IEEE/OSA/SPIE ACP 2009 Subcommittee on Network Architectures, Management and Applications (SC4), and Chair of SC4 in IEEE/OSA/SPIE ACP 2010, 2011, and 2012. She was a TPC co-chairs of IEEE/OSA/SPIE ACP 2013 and is a General co-chair of IEEE/OSA/SPIE ACP 2014. She was the general chair of IEEE International Conference on Transparent Optical Networks 2011 and Chair of the Optical Networks and Systems Symposium within IEEE ICC 2012. She was a TPC co-chair of IEEE Optical Network Design and Modeling (ONDM 2013) and is a General Chair of ONDM 2014. Professor Wosinska is a Senior Member of IEEE. 